\title{
Pourquoi s'intéresser à la douleur chronique postopératoire ?
}

\author{
G. Mick \\ (C) Springer-Verlag 2009
}

Souffrir d'une douleur chronique rebelle après un acte chirurgical peut paraitre paradoxal, en particulier pour les patients. Pour nous, praticiens et soignants confrontés quotidiennement à la problématique douloureuse chronique, cette situation bien particulière peut être perçue comme une évolution personnelle de l'individu survenant à un instant donné dans le parcours de vie, et qui s'explique foncièrement par la biographie du patient et ses interactions avec le monde médical.

La douleur chronique postopératoire est paradoxale, d'abord parce qu'elle est tout simplement consécutive à un geste a priori salvateur. Plus encore, comme on le découvre au travers de ce numéro, elle devrait être par essence prédictible, prévisible, et donc susceptible d'être prévenue. Mais cruel réalisme, il s'agit peut-être de l'un des plus beaux exemples des inégalités interindividuelles vis-àvis de la vulnérabilité, à l'échelle biologique, c'est-à-dire génétique, comme tout au long des parcours préopératoire, peropératoire et postopératoire. Et presque curieusement, la douleur chronique postopératoire peut être presque parfaitement modélisable par l'expérimentation animale : c'est par elle que la construction scientifique du concept de vulnérabilité est aujourd'hui plus que jamais à l'ordre du jour.
En clinique, la douleur chronique postopératoire pose fondamentalement la question de la transdisciplinarité, non pas tant vis-à-vis de la prise en charge du patient douloureux chronique, mais parce qu'elle procède des comportements souvent bien déterminés de nombreux acteurs avant, pendant, et après l'acte chirurgical. Au-delà de la dimension globale, s'intéresser à cette douleur impose aussi de tenter de distinguer ou d'accepter ce qui procède d'un mécanisme neuropathique, non neuropathique, mais surtout de l'interpénétration indissociable des deux.

Ce numéro propose un petit parcours initiatique de la douleur chronique postopératoire devenue aujourd'hui un défi pour tous les acteurs de santé, relevant tant de la prévention, de la coordination et de l'optimisation des soins que de l'éducation thérapeutique et de l'accompagnement. À la croisée des chemins, cette situation douloureuse chronique peut donc paraître à première vue bien particulière, mais elle peut être l'un des témoignages les plus prégnants des multiples facettes de ce que l'on appelle la douleur, de l'approche scientifique de chacune d'entre elles, mais aussi de l'approche clinique dans sa globalité, depuis la prévention jusqu'à la prise en charge symptomatique. Et dans ce domaine, pour une fois, il semble que nous ayons les moyens d'être des acteurs vraiment salvateurs. 